\title{
Central Sleep Apnea Syndrome
}

\author{
Rushika Shah ${ }^{1}$, Abha Pandey ${ }^{2}$
}

International Journal of Head and Neck Surgery (2019): 10.5005/jp-journals-10001-1367

\section{Case Description}

A 68 years male with BMI- $26 \mathrm{~kg} / \mathrm{m} 2$, known case of cerebrovascular accident 4 years ago, congestive heart failure with low left ventricular ejection fraction (LVEF) of $25 \%$ requiring diuretics since 2 years. He had complaints of excessive daytime somnolence, unrefreshing sleep and snoring during night. PSG showed evident central apneas with $\mathrm{AHI}-32 / \mathrm{hr}$ and lowest $\mathrm{SpO}_{2}-88 \%$. It also showed Cheyn-Stokes breathing pattern.

Sleep apneas have received increasing recognition since the last few years due to spread of awareness and its association with various comorbidities. Unlike obstructive sleep apnea (OSA), central sleep apneas (CSAs) are not as widely studied with evident lacuna in literature. CSA includes a variety of sleep related breathing disorders characterized by diminished or absent respiratory efforts in addition to cessation of airflow for at least 10 seconds. This is in contrast to obstructive apneas, wherein the respiratory effort is preserved and is rather paradoxical. CSA usually occurs in association with OSA or due to underlying medical ailments.

\section{Definition}

Central sleep apnea syndrome (CSAS) is defined as presence of CSA i.e period of absent airflow due to lack of respiratory efforts alongwith symptoms of snoring, excessive daytime sleepiness, morning headaches, restless sleep or insomnia. ${ }^{1}$ CSA has been classified in various ways either based on status of ventilation (hypercapnea or eucapnea) or based on causative etiology by International Classification of Sleep Disorders-32. The following criteria based on Apnea-Hypopnea index is used to grade CSA where majority of events should be central.

- Mild CSA: AHI: 5 - 15/hr

- Moderate CSA: AHI: $15-30 / \mathrm{hr}$

- Severe CSA: AHI >30/hr

\section{Pathophysiology}

Various mechanisms have been postulated for the occurrence of CSAS. However, pathophysiology can be best explained under two broad headings, i.e CSA with hyperventilation or hypoventilation (Fig. 1 and Flowchart 1).

\section{Central Sleep Apnea and Heart Failure}

Central Sleep Apnea (CSA) in heart failure (HF) needs special attention as it is found to be present in approximately $30-50 \%$ of patients with $\mathrm{HF}$ with reduced LVEF and in $18-30 \%$ of patients with preserved LVEF. ${ }^{4}$

Cheyne-Stokes breathing (CSB) is a peculiar accompaniment of patients with CSA especially those with associated heart failure.
'Pulmonary Medicine, Guru Nanak Hospital, Mumbai, Maharashtra, India

${ }^{2}$ Department of Respiratory Diseases, Lilavati Hospital and Research Centre, Mumbai, Maharashtra, India

Corresponding Author: Rushika Shah, Pulmonary Medicine, Guru Nanak Hospital, Mumbai, Maharashtra, India, e-mail: dr_rushika85@gmail.com

How to cite this article: Shah R, Pandey A. Central Sleep Apnea Syndrome. Int J Head Neck Surg 2019;10(2):34-38.

Source of support: Nil

Conflict of interest: None

CSB also known as crescendo-decrescendo pattern of breathing is defined as waxing and waning in ventilation. This disorder is most commonly observed in patients with congestive heart failure (CHF) and left ventricular systolic dysfunction. Apneas or hypopneas occur at the nadir of the characteristic crescendo-decrescendo ventilatory pattern and are most common during initial lighter sleep (stages 1 and 2). The cycle time of this pattern of unstable ventilation (typically 60 to $90 \mathrm{~s}$ ) is much longer than other forms of CSA, due to prolonged circulation time in patients with CHF. Arousal typically occurs mid-cycle at the peak of ventilatory effort rather than at the cessation of apnea. ${ }^{5}$ Recurrent events of apnea, hypoxia, arousal and re-oxygenation in HF patients with CSA have pathological consequences including sympathetic nervous system activation, oxidative stress, systemic inflammation, and endothelial dysfunction. However, there has not been robust evidence to support this but understanding of physiology definitely advocates the same.

\section{Treatment Emergent Central Sleep Apnea}

Treatment emergent CSA (TECSA) is defined as emergence of CSA (i.e central apnea index $>5 /$ hour) in patients with established OSA while they are initiated on PAP therapy, which was not present at the time of diagnosis. Prevalence of TECSA varies from 5.0 to $20.3 \%{ }^{6}$ Common risk factors identified for this entity have been male gender, advancing age, high baseline $\mathrm{AHI}$, presence of heart failure or ischemic heart disease, high baseline arousal index, high CPAP pressure and low BMI. ${ }^{7}$

Mechanism of TECSA is not well understood. Several hypotheses have been proposed as yet. It is a type of CSA only where cessation of breathing occurs when persistent $\mathrm{CO}_{2}$ washout leads to $\mathrm{PCO}_{2}$ levels below the apnea threshold due to any reason like relief of upper airway obstruction, frequent awakening due to poor sleep quality and significant mouth leak. Treatment is observation only for initial 3 months as most of TECSA events resolve in this period. For those who persist with TECSA after 3 months, assisted servo

() The Author(s). 2019 Open Access This article is distributed under the terms of the Creative Commons Attribution 4.0 International License (https://creativecommons. org/licenses/by-nc/4.0/), which permits unrestricted use, distribution, and non-commercial reproduction in any medium, provided you give appropriate credit to the original author(s) and the source, provide a link to the Creative Commons license, and indicate if changes were made. The Creative Commons Public Domain Dedication waiver (http://creativecommons.org/publicdomain/zero/1.0/) applies to the data made available in this article, unless otherwise stated. 


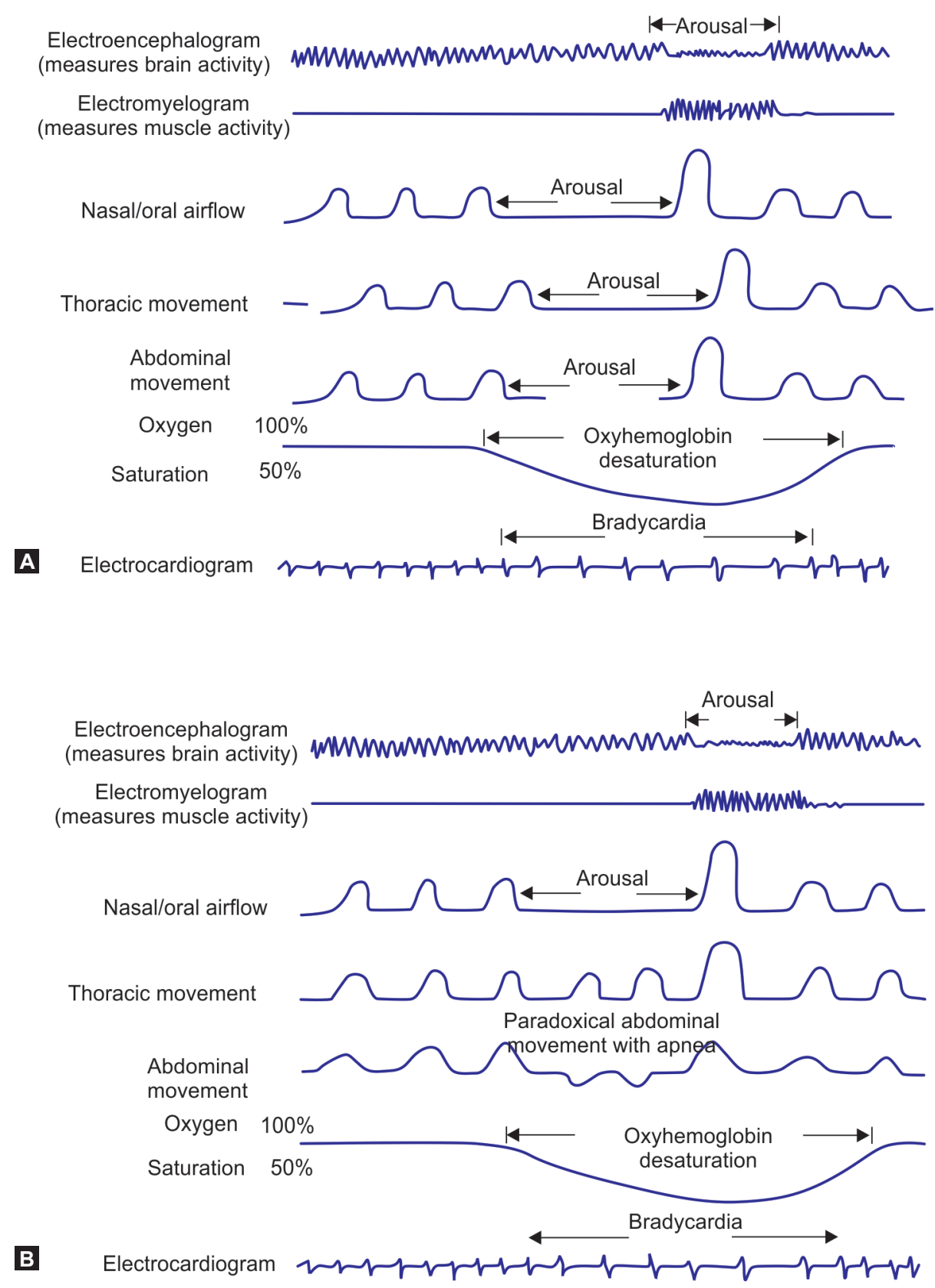

Figs $1 \mathrm{~A}$ and B: Comparison of events in obstructive and central sleep apnea on polysomnography. (A) Central event; (B) Obstructive events

ventilation (ASV) mode is preferred. All such patients should be checked for mouth leaks and over titration of PAP. Also, in a few repeat PAP titration may be needed to optimize the PAP levels. ${ }^{8}$

\section{Clinical Presentation}

Patients with CSA commonly present with poor sleep quality, insomnia, excessive daytime sleepiness, poor concentration, paroxysmal nocturnal dyspnea, morning headaches, nocturnal angina, witnessed pauses in breathing, nocturnal arrhythmias, moodiness, reduced libido and impotence. Findings of associated medical conditions should be elicited like pedal edema and raised JVP due to heart failure and asymmetric weakness due to stroke, etc. The clinical characteristics vary slightly between hypercapnic and nonhypercapnic central sleep apnea (Tables 1 to 3).

\section{Risk Factors for CSA}

- Age: Adults $>65$ years

- Gender: Males > Females

- Heart failure: Males $>60$ years, atrial fibrillation, daytime $\mathrm{PaCO}_{2}$ $<38 \mathrm{~mm}$ of $\mathrm{Hg}$

- Stroke: acute events and usually self-limiting

- Miscellaneous: acromegaly, renal failure

- Medications: opioids

\section{DiAgnOSIS}

Any patient with the presence of daytime sleepiness with presence of any risk factor or $>1$ symptom or sign of CSA should be subjected to a full night, attended, in-lab polysomnography which is the gold standard for diagnosis of CSA. Essentially, level 1 polysomnography 
Flowchart 1: Schematic diagram of events leading to central sleep apnea ${ }^{3}$

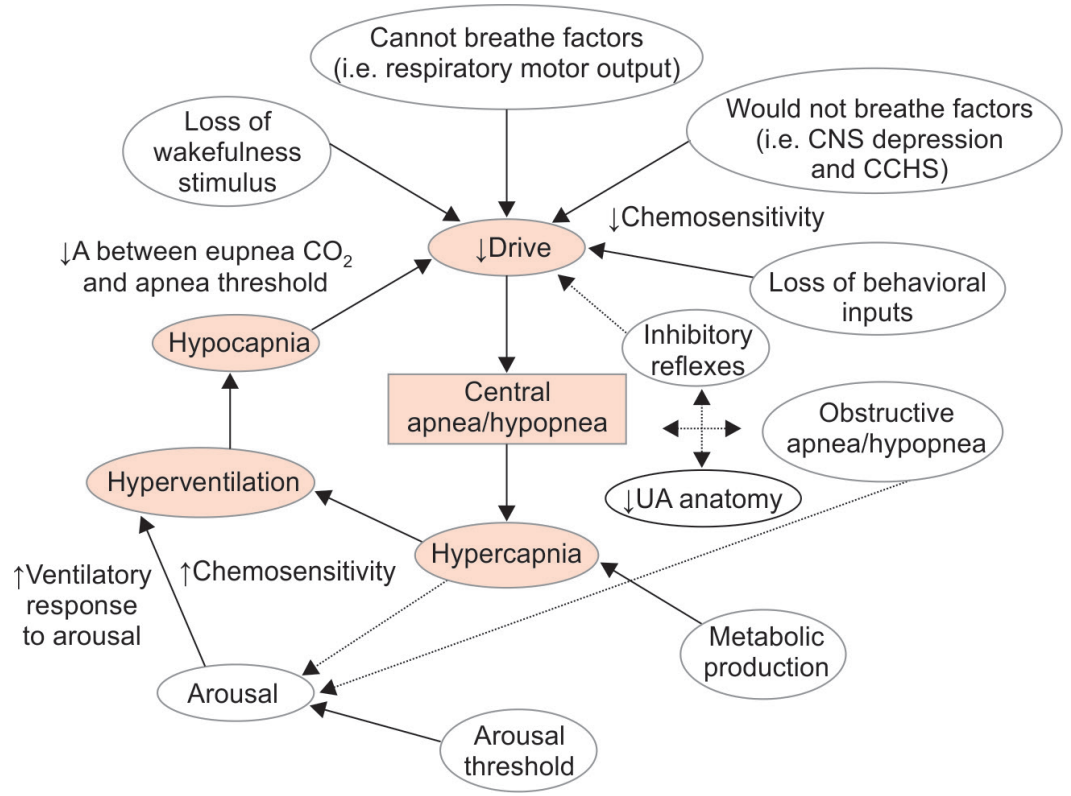

Table 1: Classification of central sleep apnea ${ }^{1}$

\begin{tabular}{ll}
\hline Hypercapnic $\left(\mathrm{PCO}_{2}>45\right)$ & Nonhypercapnic $\left(\mathrm{PCO}_{2} \leq 45\right)$ \\
\hline Central alveolar hypoventilation & a. Secondary \\
a. Secondary & Congestive heart failure $(\mathrm{CHF})$ \\
Brainstem tumors & Brain lesions \\
Bulbar polio & Renal failure \\
Encephalitis & Acromegaly \\
b. Primary & Cerebrovascular disease \\
Respiratory neuromyopathy & Atrial fibrillation \\
Neuromyopathies & High altitude periodic breathing \\
Myotonic dystrophy & Opioid related \\
Muscular dystrophy & Complex sleep apnea \\
Myasthenia gravis & b. Primary \\
Amyotrophic lateral sclerosis & Idiopathic central sleep apnea \\
Postpolio syndrome & \\
Diaphragm paralysis & \\
\hline
\end{tabular}

Table 2: Pathophysiology and consequences of CSAS

\begin{tabular}{ll}
\hline Hyperventilation & Hypoventilation \\
\hline Posthypocapnia response to restore $\mathrm{CO}_{2}$ & $\begin{array}{l}\text { - Removal of wakefulness stimulus to breathe } \\
\text { - Compromised neuromuscular ventilator control }\end{array}$ \\
Seen in CHF, high altitude CSA, primary CSAS, etc & $\begin{array}{l}\text { Seen in CNS disorders like encephalitis, neuromuscular disorders, kyphoscoliosis, } \\
\text { etc. }\end{array}$ \\
Leads to & Leads to \\
- Hypocapnia in awake or sleep state & - Severely reduced ventilatory output \\
- Sleep state instability & - Insufficient alveolar ventilation \\
- Chronic hyperventilation state & - Chronic hypoventilation state \\
\hline
\end{tabular}

CHF, chronic heart failure; CNS, central nervous system

is required to diagnose a CSA as it can detect respiratory effort and airflow limitation. As a general rule, patients with glaring cardiac, neurological comorbidities and also those who are on codeine preparations for considerable period of time, complete overnight polysomnography should be opted than a limited channel study as they are much more predisposed for central events.
Diagnostic criteria varies as per the type of CSA like CSA associated with Cheyne-Stokes breathing, high altitude or primary CSA. Importantly, all patients should have symptoms of EDS, nocturnal awakening, snoring, witnessed apneas or insomnia with polysomnography showing $\geq 5$ central apnea (CA) and /or central hypopnea $(\mathrm{CH})$ in an hour of sleep; the number of $\mathrm{CA}$ and/or $\mathrm{CH}$ is 
Central Sleep Apnea Syndrome

Table 3: Clinical characteristics of patients with central sleep apnea ${ }^{1}$

\begin{tabular}{lll}
\hline Characteristic & Hypercapnic & Nonhypercapnic \\
\hline Sex distribution & Equal & Predominantly male \\
History of respiratory failure & Frequent & Not reported \\
Peripheral edema and cor pulmonale & Frequent & Not reported reported \\
Polycythemia & Frequent & Not reported \\
Muscle weakness & Frequent & Uncommon \\
Morning headaches & Common & Frequent \\
Snoring & Common & Common \\
Nasal obstruction & Uncommon & Common \\
Hypertension & Uncommon & Common \\
Nocturnal choking & Uncommon & Common \\
Nocturnal awakenings and insomnia & Uncommon & Frequent \\
Excessive daytime sleepiness & Frequent & Common \\
Restless sleep & Common &
\end{tabular}

$>50 \%$ of the total no of apneas and hypopneas. Along with these features supportive of accompanying etiology should be present. Disorder is not better explained by another current sleep disorder, medical or neurological disorder, medication use or substance use disorder. Also, congenital central alveolar hypoventilation or Ondine's curse should be ruled out.

In our case patient is elderly male, with underlying risk factors for CSA like heart failure and cerebrovascular accident in past. PSG showed evident central apnea events with Cheyne-Stokes breathing, classical of CSA.

\section{Differential Diagnosis}

Obstructive sleep apnea periodic limb movement disorder, Rotating Shift Workers, Narcolepsy, respiratory diseases such as COPD, ILD, asthma, neuromuscular disorder should be ruled out.

\section{TREATMENT}

Goals of therapy in CSA are mainly directed to achieve a normal sleeping pattern thus reducing the daytime sleepiness and improving the quality of life. Patients with mild-to-moderate CSA with minimal symptoms can be put on management of underlying etiology. If CSA persists despite the best management, CSA specific therapy like positive airway pressure therapy should be initiated. However, in patients with severe CSA, positive airway pressure therapy should be initiated parallel to management of underlying etiology.

\section{Positive Airway Pressure Therapy \\ Continuous Positive Airway Pressure (CPAP)}

Initial small trials in CSA patients showed that CPAP could improve the ejection fraction of heart and the quality of life. This method of treatment was associated with reduction in $\mathrm{AHI}$, nocturnal urinary frequency, daytime plasma norepinephrine levels and ventricular ectopic beats.

\section{Bilevel PAP Therapy}

Bilevel PAP therapy along with maintaining a pneumatic splint improves alveolar ventilation. Role of bilevel PAP therapy is in those CSA patients who are not tolerating CPAP well, have evident hypoventilation pattern persistent despite CPAP therapy. Back up rate should always be set to avoid any incipient periodic breathing.

\section{Assisted Servo-Ventilation}

Ideal mode of ventilation for CSA would the one which adapts to the breathing rate and tidal volume of a patient and matches it at the end of minute ventilation. ASV mode supplies a small sustained positive air pressure. It senses an event of CSA and appropriately delivers the tidal volume at a preset respiratory rate (set in the device). The goal of assisted servo-ventilation (ASV) is to maintain a precise ventilation with tight control of $\mathrm{PaCO}_{2}$ by avoiding ventilatory overshoot and undershoot which in turn dampens periodic breathing cycle. It has been found to improve LVEF, quality of life and overall sleep quality. ASV mode is indicated in patients with associated $\mathrm{HF}$ and preserved $\mathrm{EF}>45 \%$.

\section{Pharmacologic Therapy}

As an alternative to CPAP therapy when patients are not benefitting or tolerating the same, pharmacological therapy can be tried. It includes drugs like acetazolamide and theophylline. Acetazolamide acts by causing metabolic acidosis which stimulates the respiratory centre thus abolishing the resultant apneas. It has found some benefit in patients with hyperventilation related CSA. Theophylline has been found to have some benefit in patients with CSA associated with CSB due to heart failure. It acts as a respiratory stimulant and can reduce the AHI. However, both these drugs carry side effects and hence should be used in the right patient type with caution. Recently, zolpidem and triazolam have been considered as an option for treatment of primary CSAS, if the patient does not have any underlying risk factors for respiratory depression.

\section{Adjuvant Therapy}

Oxygen therapy can be used as a salvage therapy if patient doesn't tolerate CPAP at all especially in those with associated heart failure. Bicarbonate buffers during night hour dialysis can be used in CSA patients with accompanying renal failure.

\section{Points to Remember}

- CSA is an important accompaniment of OSA, often underdiagnosed

- CSA should be suspected in patients with suggestive symptoms and underlying neurological disorders, heart failure, long-term opioids usage and those with OSA on PAP therapy.

- Diagnosis ofCSA warrants a supervised level I polysomnography 
- Identification of cheynes stokes breathing is of great importance in CSA

- Depending upon the state of ventilation PAP therapy should be initiated in patients

\section{References}

1. Iber C, Ancoli-Israel S, et al. The AASM Manual for the Scoring of Sleep and Associated Events: Rules, Terminology and Technical Specification, 1st ed. Westchester, IL: American Academy of Sleep Medicine, 2007.

2. American Academy of Sleep Medicine (2014). The International Classification of Sleep Disorders, Third Edition (ICSD)3.

3. Danny J. Eckert etal. Central Sleep Apnea Chest. 2007 February; 131(2):595-607.
4. Oldenburg O, Lamp B, et al. Sleep disordered breathing in patients with symptomatic heart failure: a contemporary study of prevalence in and characteristics of 700 patients. Eur JHeart Fail 2007;9:251-257.

5. Trinder J, Merson R, et al. Pathophysiological interactions of ventilation, arousals, and blood pressure oscillations during CheyneStokes respiration in patients with heart failure. Am J Respir Crit Care Med 2000;162:808-813.

6. Mador MJ. Emergent central sleep apnea during CPAP therapy-clinical implications. J Thorac Dis 2017;9(11):4182-4184.

7. Nigam G, Pathak C, et al. A systematic review on prevalence and risk factors associated with treatment- emergent central sleep apnea. Ann Thorac Med 2016;11;202-210.

8. Dellweg D, Kerl J, et al. Randomized controlled trial of noninvasive positive pressure ventilation (NPPV) versus servoventilation in patients with CPAP-induced central sleep apnea (complex sleep apnea). Sleep. 2013;36(8):1163-1171. 